\title{
Monitoring of Patient-Ventilator Interaction at the Bedside
}

\author{
Marjolein de Wit MD MSc
}

\author{
Introduction \\ Trigger Asynchrony \\ Flow Asynchrony \\ Cycling Asynchrony \\ Summary
}

\begin{abstract}
Monitoring of patient-ventilator interactions at the bedside involves evaluation of patient breathing pattern on ventilator settings. One goal of mechanical ventilation is to have ventilator-assisted breathing coincide with patient breathing. The objectives of this goal are to have patient breath initiation result in ventilator triggering without undue patient effort, to match assisted-breath delivery with patient inspiratory effort, and to have assisted breathing cease when the patient terminates inspiration, thus avoiding ventilator-assisted inspiration during patient exhalation. Asynchrony can occur throughout the respiratory cycle, and this paper describes common asynchronies. The types of asynchronies discussed are trigger asynchrony (ie, breath initiation that may manifest as ineffective triggering, double-triggering, or auto-triggering); flow asynchrony (ie, breath-delivery asynchrony, which may manifest as assisted-breath delivery being faster or slower than what patient desires); and cycling asynchronies (ie, termination of assisted inspiration does not coincide with patient breath termination, which may manifest as delayed cycling or premature cycling). Various waveforms are displayed and graphically demonstrate asynchronies; basic principles of waveform interpretation are discussed. Key words: mechanical ventilation; monitoring; patient-ventilator interaction. [Respir Care 2011;56(1):61-68. () 2011 Daedalus Enterprises]
\end{abstract}

\section{Introduction}

Monitoring of patient-ventilator interactions at the bedside is an integral part of caring for the critically ill patient. Caring for the mechanically ventilated patient involves examining the impact of patient breathing and behavior on ventilator settings, and vice versa. An uncomfortable-ap-

\footnotetext{
Marjolein de Wit MD MSc is affiliated with the Division of Pulmonary Disease and Critical Care Medicine, Department of Internal Medicine, Virginia Commonwealth University, Richmond, Virginia.

The author has disclosed no conflicts of interest.

Dr de Wit presented a version of this paper at the 46th RESPIRATORY CARE Journal Conference, "Patient-Ventilator Interaction," held March 19-21, 2010, in Cancún, Quintana Roo, Mexico.

Correspondence: Marjolein de Wit MD MSc is affiliated with the Division of Pulmonary Disease and Critical Care Medicine, Department of Internal Medicine, Virginia Commonwealth University, Post Office Box 980050, Richmond VA 23298-0050. E-mail: mdewit@mcvh-vcu.edu.
}

pearing patient should not only be evaluated for physical and psychological causes of distress, but clinicians should make sure that ventilator settings are optimal. The aim of this paper is to serve as a resource and aid clinicians, including respiratory therapists, residents, fellows, and hospitalists who are gaining exposure to the mechanically ventilated patient. Many excellent texts have been written on this topic. ${ }^{1-4}$ The aim here is to serve as an introductory resource and aid inexperienced clinicians in understanding the basics of patient-ventilator interactions.

Accurate assessment of patient-ventilator interactions and work of breathing (WOB) requires invasive measurements of pleural pressure and/or respiratory muscle electromyogram. Use of an esophageal balloon, which permits determination of pleural pressure, and respiratory muscle electromyograms have been used to measure a variety of patient-ventilator interactions and to compute WOB. ${ }^{5-9}$ However, these devices are not used during routine patient care, and clinicians must rely on physical examination of the patient as well as visual inspection of waveforms to assess for patient-ventilator syn- 
chrony and asynchrony. Visual inspection of waveforms has been shown to correlate well with esophageal-balloon readings, but is not without error. ${ }^{5,6}$ Artifacts such as cardiac oscillation may mimic asynchronies, and clinicians must learn to distinguish between these and true asynchronies. ${ }^{10}$ There are times when clinicians standing at the bedside are unable to distinguish between asynchrony and artifact with certainty, in which case he/she has to use clinical judgment to determine optimal patient treatment.

Patients on mechanical ventilation may perform no WOB or some WOB. In addition, in some situations, patients experience increased WOB. The increased WOB generally occurs when either the ventilatory support to overcome the resistance of the ventilator circuit and endotracheal tube is inadequate or when the patient is not in synchrony with the ventilator, as for example when the patient is exhaling during ventilator-assisted inhalation. Patient WOB can be computed knowing pleural pressure, but this information is not readily available in daily practice. Therefore, the clinician must make inferences about patient WOB and response to ventilator settings. Careful observation of the patient provides information about WOB. Facial expressions, accessory muscle use, active exhalation, and "mouth breathing" provide information about patient comfort and distress.

It is important for clinicians not to assume that ventilator settings are optimal for the patient. Rather, clinicians must evaluate the patient and response to ventilator settings before drawing conclusions about patient-ventilator synchrony. The patient is the focus point, and the clinician must adjust the mechanical ventilator to meet the patient's ventilatory requirements. The goal is to have the "right tool for the right job," and clinicians must not assume that one "tool" (ie, set of ventilator parameters) satisfies the needs of different patients. It is only after careful observation of the patient and examination of ventilator waveforms that clinicians should assume the patient and ventilator are synchronous. When a patient appears uncomfortable, physical examination and evaluation of ventilator waveforms are the first steps in the management of the patient. This assessment provides useful clinical information and often permits rapid correction of patient distress. In addition, sedation and neuromuscular blocking agents should not be used routinely to improve patient-ventilator synchrony. These agents should be used only after clinicians have optimized synchrony, and depression of the patient's respiratory effort is still required.

Modern ventilators display real-time information in the form of waveforms. Common waveforms are pressure-time, flow-time, and volume-time (Figs. 1 and 2). A ventilatorassisted breath can be divided into various parts: breath initiation, breath delivery, breath termination, and mechanical exhalation. Mechanical exhalation refers to the period of time when the patient is not receiving ventilator-assisted inspiration. The goal of patient-ventilator synchrony is to have the various parts of ventilator-assisted breathing coincide with

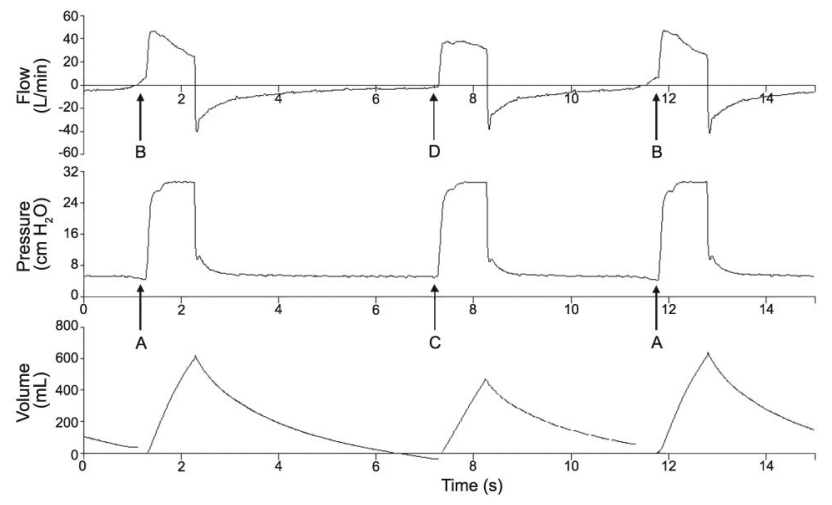

Fig. 1. Examples of flow-time, pressure-time, and volume-time waveforms displayed on modern ventilators. Positive-pressure mechanical ventilation increases airway pressure during inspiration. Air flow into the thorax is marked by positive deflections on the waveforms. The first and third breaths are triggered by the patient, and these breaths demonstrate a pressure decrease (arrow A) and a flow increase (arrow B) prior to ventilator-assisted-breath delivery. The second breath is not triggered by the patient and is initiated by the ventilator: no negative deflection (arrow C) or flow increase (arrow D) is noted at the beginning of the breath. In this example, the 3 breaths have the same peak airway pressure but different flow waveforms and tidal volume. This indicates a standard pressure-control mode and not a volume-control mode or a volume-targeted pressure-control mode. The fact that all 3 breaths have identical inspiratory time indicates time-cycling, thus each breath is mandatory (ie, ventilator-cycled) and not spontaneous (ie, patient-cycled). ${ }^{11}$ The fact that patient-triggered breaths are mandatory indicates the breath sequence is continuous mandatory ventilation. Thus, the mode is pressure-control continuous mandatory ventilation, as opposed to, for example, volume-control intermittent mandatory ventilation or pressure support. The inspiratory pressure (ie, the change in airway pressure driving inspiratory flow) is peak airway pressure minus PEEP, or approximately $29 \mathrm{~cm} \mathrm{H}_{2} \mathrm{O}-5 \mathrm{~cm} \mathrm{H}_{2} \mathrm{O}=24 \mathrm{~cm} \mathrm{H}_{2} \mathrm{O}$.

the patient's intrinsic breathing pattern. Breath initiation is called triggering and may be performed by the patient or by the ventilator (see Fig. 1). When a patient triggers the ventilator, the patient increases intrathoracic volume, which results in the decrease in airway pressure and increase in air flow. If the pressure (called pressure-triggering) or air flow (called flow-triggering) reaches the threshold set by the clinician, then the ventilator delivers a breath. Because there is a time delay between the patient's action and the ventilator response, the pressure-time waveform demonstrates a transient decrease in airway pressure and a transient increase in air flow (see Figs. 1 and 2).

Breath delivery is achieved by targeting either a flow (flowtargeted breath) or a pressure (pressure-targeted breath). In this paper, I will consider flow-targeted breaths in which the clinician manually sets the flow, and flow ceases once the preset tidal volume $\left(\mathrm{V}_{\mathrm{T}}\right)$ has been delivered. This review will consider 2 types of pressure-targeted breaths. One type is called pressure support; it is always spontaneous (ie, patienttriggered and patient-cycled, as in pressure support). ${ }^{11}$ The 


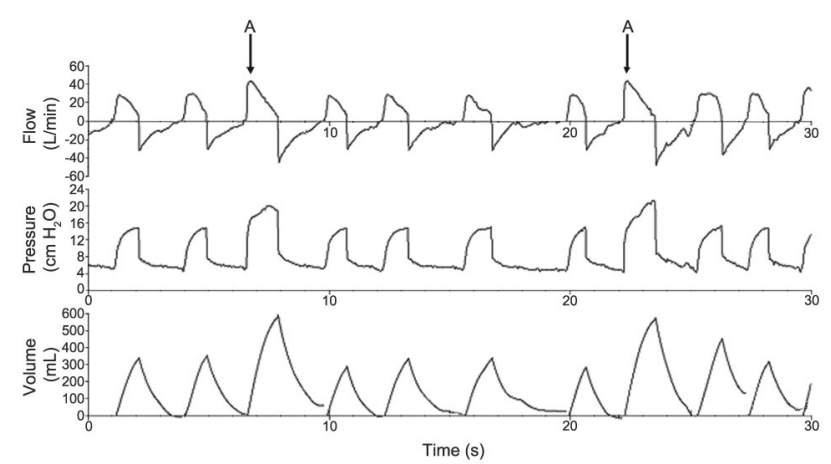

Fig. 2. This tracing depicts 30 seconds of information. Careful review of the waveforms reveals 2 types of breaths. One type of breath (arrow A) has the same peak flow, flow waveform (decelerating), and tidal volume, but different peak inspiratory pressures. This indicates volume-control mandatory breaths. The second type shows consistent (lower) peak airway pressures but different volumes and flows, indicating these breaths are pressure-targeted breaths. Furthermore, these breaths have random start times and variable inspiratory times, indicating that they are patient-triggered and patient-cycled and are thus spontaneous breaths. They are in fact pressure-support breaths. Therefore, we conclude that the mode is volume-control intermittent mandatory ventilation with pressure support. The PEEP is $5 \mathrm{~cm} \mathrm{H}_{2} \mathrm{O}$.

second type is mandatory (ie, machine-triggered and/or machine-cycled, as in assist control). ${ }^{11}$ The speed at which the targeted pressure is reached is called the rise time. Flow can be mapped over time by visually inspecting the flow-time waveform. In these graphs, positive flow is inhalation while negative flow is exhalation (see Figs. 1 and 2).

Cycling is defined as termination of inspiration and is achieved when either a preset volume has been delivered (volume-control breath), a preset time has been reached (pressure-targeted, time-cycled breath), or a preset flow has been reached (flow-cycled breath). Globally, most patients are ventilated using volume-control breaths, and the flow parameters are set by the clinician. ${ }^{12}$ In a pressure-targeted breath with a fixed inspiratory time (sometimes referred to as pressurecontrol), the pressure is applied for a period of time determined by the clinician; thus, all breaths have the same ventilator-assisted inspiratory time. Pressure-support breaths, on the other hand, have variable ventilator-assisted inspiratory times. A pressure-support breath is terminated when flow reaches a preset proportion of the peak flow (see Fig. 2), which is called the cycling sensitivity. For example, if a patient has a peak flow of $60 \mathrm{~L} / \mathrm{min}$ and the cycling sensitivity is set to $25 \%$, then assisted inspiration is halted when flow reaches $15 \mathrm{~L} / \mathrm{min}$.

\section{Trigger Asynchrony}

Trigger asynchronies discussed here are ineffective triggering, double-triggering, and auto-triggering. Ineffective triggering and double-triggering are the most common asyn- chronies in patients undergoing invasive mechanical ventilation, accounting for at least $80 \%$ of asynchronous breaths. ${ }^{13}$ Auto-triggering accounts for $<2 \%$ of asynchronies. ${ }^{13,14}$

A quarter of patients experience ineffective triggering in at least $10 \%$ of breaths. ${ }^{13,14}$ Ineffective triggering is associated with increased duration of mechanical ventilation in intensive care unit patients. ${ }^{13,14}$ In patients undergoing mechanical ventilation in long-term acute-care units, ineffective triggering is associated with decreased likelihood of successful weaning. ${ }^{5}$ It is unclear if ineffective triggering increases the severity of illness and the duration of mechanical ventilation or is a marker of greater severity of illness. Factors associated with ineffective triggering are improper triggering threshold, airtrapping (auto-PEEP), muscle weakness, decreased respiratory drive, and deeper level of sedation. ${ }^{5,13-18}$ Thus, patients with ineffective triggering may not appear agitated. Due to depth of sedation, some patients with ineffective triggering may not appear to be asynchronous, unless the clinician attempts to identify the problem by carefully examining the patient and ventilator waveforms.

When the patient attempts to initiate a breath but the trigger threshold is not reached, then the breath is ineffectively triggered. Ineffective triggering is marked by a decrease in pressure of $\geq 0.5 \mathrm{~cm} \mathrm{H}_{2} \mathrm{O}$ associated with a simultaneous increase in air flow. ${ }^{13}$ Insensitive triggering should be avoided because ineffective triggering increases patient WOB and may result in respiratory muscle fatigue as well as delayed triggering. Flowtriggering may be more responsive to patient needs than is pressure-triggering. ${ }^{19}$

Ineffectively triggered breaths are not included on the ventilator display of the respiratory rate. The ventilator alarm does not sound, and clinicians can only detect ineffective triggering by evaluation of ventilator waveforms. Examination of the patient experiencing ineffective triggering is marked by expansion of the patient's thoracic volume without accompanied delivery of assisted ventilator breath. When the clinician places his/her hand on the patient's thorax, he/she will notice increasing thoracic volume without delivery of ventilator breath. Ineffective triggering is manifested graphically by a decrease in airway pressure associated with a simultaneous increase in air flow. Figures 3 and 4 display examples of ineffective triggering.

Ineffective triggering is of particular importance in patients with COPD. 5, 17,18,20 Patients with COPD have prolonged expiratory times, and the patient's expiratory time may be inadequate in relation to ventilator settings. When a patient with COPD receives a large ventilator-assisted breath, the time to exhale is insufficient, resulting in air-trapping and auto-PEEP; the ventilator does not detect auto-PEEP. This increases the likelihood that the patient may not reach the trigger threshold, resulting in ineffective triggering. Increasing the breath size increases the likelihood of ineffective trig- 


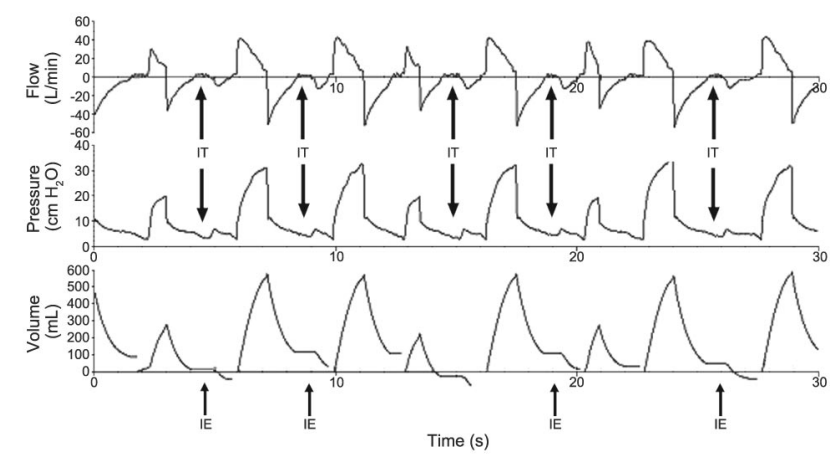

Fig. 3. During this tracing of 30 seconds, the ventilator displays that the patient rate is 16 breaths $/ \mathrm{min}$. However, in the time period of 30 seconds the patient has 5 attempts that do not result in triggering of the ventilator (ineffective triggering, arrows IT). The actual patient respiratory rate is 26 breaths $/ \mathrm{min}$. In this example, most of the ineffectively triggered breaths are marked by incomplete exhalation (arrows IE; note that the inhaled volume is larger than the exhaled volume), which may suggest that air-trapping is the cause of ineffective triggering. Placement of an esophageal balloon permits certain determination of auto-PEEP but this device is not used as part of routine patient care. The clinician must make inferences about the cause of ineffective triggering. Note that there are 2 types of breaths: one set with peak pressure $19 \mathrm{~cm} \mathrm{H}_{2} \mathrm{O}$, and a second set with peak pressure $30 \mathrm{~cm} \mathrm{H}_{2} \mathrm{O}$. One type of breath has the same tidal volume but different peak inspiratory pressures, indicating that the breaths are volume-control mandatory breaths. The second type of breath shows consistent (lower) peak airway pressures but different volumes and flows, indicating pressure-targeted breaths. Furthermore, these breaths have random start times and inspiratory times, indicating that they are patient-triggered and patient-cycled, and are thus spontaneous breaths. Therefore, we conclude that the mode is volumecontrol intermittent mandatory ventilation with pressure support. PEEP is $5 \mathrm{~cm} \mathrm{H}_{2} \mathrm{O}$.

gering. ${ }^{18}$ When a patient has a pressure-targeted breath of at least $20 \mathrm{~cm} \mathrm{H}_{2} \mathrm{O}$ above PEEP, then half the breaths are likely to be ineffectively triggered, and the respiratory rate displayed on the ventilator will be inaccurate. ${ }^{18}$ When the breath size is decreased, air-trapping diminishes and the patient can more readily reach the trigger threshold, increasing the rate displayed on the ventilator. ${ }^{16}$ The clinician should not assume that patient is intolerant of smaller breaths. Rather, the patient rate is likely to be the same, and previous ineffective efforts are now resulting in triggered breaths. Examination of the patient is crucial: should the patient not appear uncomfortable, then it is reasonable to assume that the smaller breath size is adequate.

Double-triggering occurs when a patient's inspiratory effort continues throughout the preset ventilator inspiratory time and remains present after ventilator inspiratory time has finished. The patient's inspiratory effort is strong enough to trigger another breath. Thus, the patient receives, in effect, a double $\mathrm{V}_{\mathrm{T}}$, with its attendant risk of lung over-inflation. This type of asynchrony may be due to unusually high ventilatory demand on the part of the patient or an inappropriate cycle

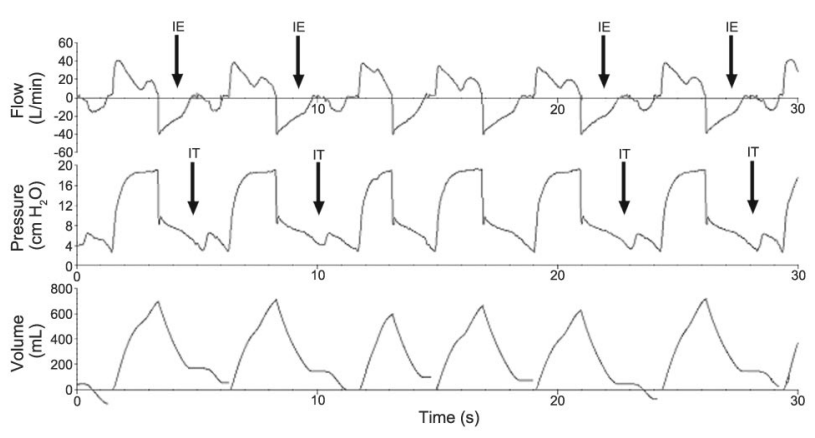

Fig. 4. In this tracing of 30 seconds, 4 breaths are ineffectively triggered (arrows IT) and 7 are effectively triggered. The rate displayed on the ventilator is 14 breaths/min, but the actual rate is 22 breaths/min. Note that the ineffectively triggered breaths occur while exhalation is incomplete (flow is not zero, arrows IE), suggesting that air-trapping may be the cause of ineffective triggering.

threshold set by the operator (eg, $\mathrm{V}_{\mathrm{T}}$ too small, inspiratory time too short, or flow-cycle threshold too high).

Auto-triggering occurs when the ventilator delivers an assisted breath that was not initiated by the patient. Clinicians may intentionally set ventilator parameters such that a patient does not trigger the ventilator, as for example when wishing to completely offload respiratory muscles and wishing to minimize oxygen consumption. However, auto-triggering may also be due to artifacts such as cardiac oscillations or inappropriately sensitive triggering thresholds. ${ }^{10}$

\section{Flow Asynchrony}

Flow asynchrony may be due to ventilator flow being either too fast or too slow for the patient, and may occur with either flow-targeted breaths or with pressure-targeted breaths. In flow-targeted breaths the clinician typically chooses the speed of the flow and the pattern of the flow. The pattern of flow may be constant over time (square waveform on the flow-time waveform), decelerating over time (peak flow is at the beginning of ventilator-assisted inspiration and is called a descending ramp, see Fig. 2 arrow A), accelerating over time (peak flow is maximal at end of assisted inspiration and is called ascending ramp), or sinusoidal over time (peak flow is in the middle of assisted inspiration). In a pressure-targeted breath the speed at which the targeted pressure is reached is dependent on the rise time, with faster rise times resulting in higher flows and shorter duration to achieve the pressure set by the clinician.

In order to unload respiratory muscles, a patient should not be required to perform excessive WOB, and may be required to perhaps only trigger the ventilator. However, it is important to note that respiratory muscle contraction does not terminate after ventilator triggering. ${ }^{21}$ The aim of achieving flow synchrony is to limit WOB and to avoid respiratory muscle 


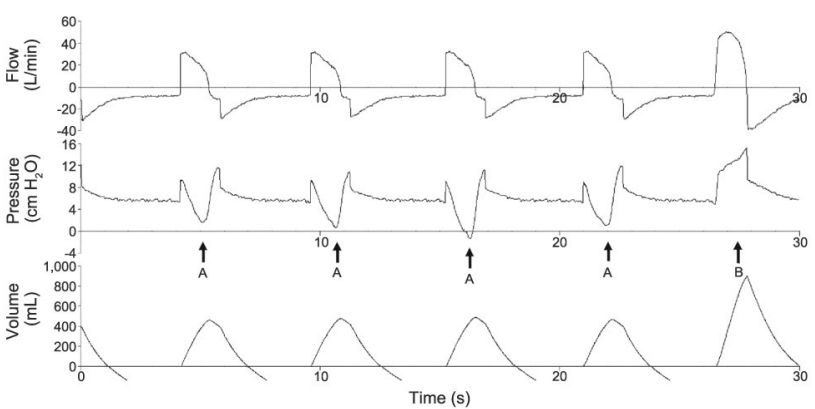

Fig. 5. Even though this patient is undergoing positive-pressure mechanical ventilation, the first 4 breaths have a relatively negative pressure (ie, pressure is below PEEP, arrows A). One breath (third breath) even has an absolute negative pressure (ie, below atmospheric pressure). Only the last breath (arrow B) does not have a relatively negative pressure during ventilator-assisted inspiration. The peak flow of the breaths with relatively negative pressure (arrows A) during assisted inspiration is lower that the peak flow of the breath that is positive throughout mechanical inspiration (arrow B). The breath size of the last breath (arrow B) is also larger than all the other breaths. The breaths marked by arrows $A$ have the same flow and the same tidal volume; these breaths are flow-targeted (peak flow $35 \mathrm{~L} / \mathrm{min}$ ) with descending ramp and volume-control $(500 \mathrm{~mL})$. The patient is ventilated with intermittent mandatory ventilation with volume control, flow-targeted ventilation, and pressure support. The last breath (arrow B) has a pressure support of $7 \mathrm{~cm} \mathrm{H}_{2} \mathrm{O}$ and the peak flow measured is $55 \mathrm{~L} / \mathrm{min}$. The PEEP is $6 \mathrm{~cm} \mathrm{H}_{2} \mathrm{O}$. Note that the volume-control breaths are not patient initiated; only the pressure-support breath (arrow B) is patient initiated. Flow in the volume-control breaths (arrow A) has not returned to zero prior to breath initiation.

fatigue. When patient respiratory drive is elevated, the pressure-time waveform may reveal a dip during assisted inspiration. This dip occurs when the ventilator flow is below the patient's desired flow, and the patient "pulls down" the pressure-time waveform during assisted inspiration. The equation of motion summarizes this interaction between ventilator pressure and patient respiratory muscle effort:

$$
\mathrm{P}_{\text {vent }}+\mathrm{P}_{\text {mus }}=\dot{\mathrm{V}} \times \mathrm{R}+\mathrm{V} \times \mathrm{E}
$$

where $\mathrm{P}_{\mathrm{vent}}$ is ventilator pressure, $\mathrm{P}_{\mathrm{mus}}$ is muscle pressure, $\dot{V}$ is flow, $R$ is resistance, $V$ is volume, and $E$ is elastance. Because volume-control breaths have preset peak flow and flow pattern (ie, square, decelerating, accelerating, or sinusoidal), the problem with flow asynchrony may be particularly common in volume-control breaths. In pressuretargeted breaths, flow is variable and thus more responsive to patient needs.

Figure 5 is from a patient with septic shock who was being ventilated with volume-control intermittent mandatory ventilation and pressure support. The peak flow is set to $35 \mathrm{~L} /$ min in the volume-control breath. In the pressure-support breaths the target is a peak pressure; the flow is variable and set to achieve the peak pressure. In this particular patient, the peak flow was $55 \mathrm{~L} / \mathrm{min}$ during the pressure-support breath,

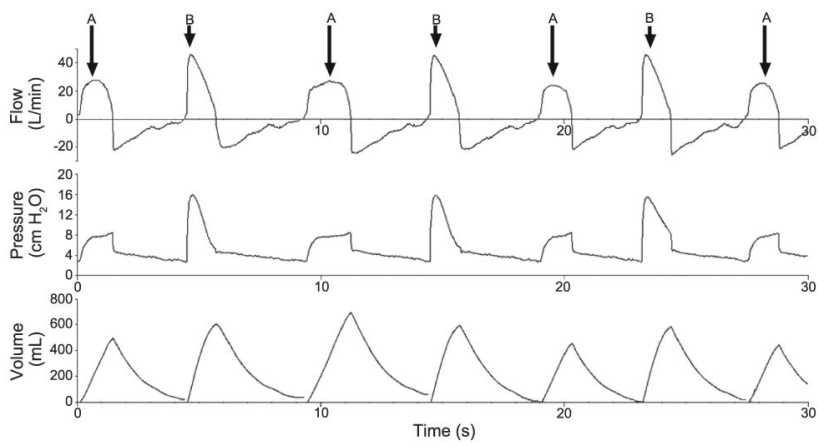

Fig. 6. This tracing displays 2 breath types with distinct maximum flows and peak airway pressures. The breaths with the lower peak flows have a lower peak airway pressure (arrows A). The breaths denoted by arrows $A$ have different tidal volumes (as seen in breaths 3 and 7), the same peak pressure, but different peak flows (albeit all are $<30 \mathrm{~L} / \mathrm{min}$, as seen in breaths 3 and 5), and different durations (breaths 3 and 5). These breaths are all patient-triggered and are pressure-support breaths. Breaths denoted by arrow $B$ have the same peak flow ( $45 \mathrm{~L} / \mathrm{min}$ ), the same flow pattern (descending ramp), and the same tidal volume $(600 \mathrm{~mL})$, but different pressure morphologies, indicating that the breaths are volumecontrol, flow-targeted breaths. The mode is intermittent mandatory ventilation with pressure support. The rate set by the clinician is 6 breaths $/ \mathrm{min}$, and the patient rate is 14 breaths $/ \mathrm{min}$. This tracing also displays delayed cycling, as marked by tenting at the end of the pressure-support breaths (arrows A), which can be corrected by changing the cycling sensitivity.

$20 \mathrm{~L} / \mathrm{min}$ higher than in the flow-targeted, volume-control breath. The patient desires a higher peak flow and is generating relatively negative intrathoracic pressures, resulting in a downward deflection of the pressure-time waveform during volume-control breaths. In this example the pressure is negative with respect to PEEP. Typically, these patients appear air-hungry: they may be using accessory muscles and may be gasping for air. This problem can be corrected by increasing peak flow in the volume-control breaths to meet the patient's desired flow. As the clinician manually increases the flow, the downward deflection in the pressure-time waveform may be observed to improve. If the flow pattern is not deceleration, consideration should be given to changing flow pattern to decelerating (ie, peak flow is at the beginning of ventilatorassisted inspiration), as typically peak air flow is at the beginning of the inspiratory cycle. In some patients, increasing the peak flow does not ameliorate the problem. These patients typically have a very high minute ventilation and may benefit from being ventilated with a pressure mode, thus targeting a pressure and allowing for variable flow. ${ }^{22}$

When ventilator flow is faster than the patient's respiratory drive, the pressure-time waveform reveals a peaking of airway pressure at the beginning of inspiration, in cases where the flow pattern is decelerating (ie, peak air flow is at the beginning of assisted inspiration). Figure 6 is from a patient intubated because of upper-airway obstruction from a thyroid mass. This patient was ventilated with volume-control inter- 
mittent mandatory ventilation and pressure support. The peak flow in the volume-control breaths is higher than that desired by the patient, resulting in peaking of the pressure at the beginning of the assisted breath. The pressure-support breaths have a lower maximum flow than the volume-control breaths. In the pressure-support breath the peak flow is $<30 \mathrm{~L} / \mathrm{min}$, whereas in the volume-control breaths the peak flow is $45 \mathrm{~L} /$ min. In this patient a flow of $45 \mathrm{~L} / \mathrm{min}$ was too high and resulted in peak pressure occurring at the beginning of the breath. It also resulted in a significantly higher peak pressure than the pressure-support breath, even though the $\mathrm{V}_{\mathrm{T}}$ of the second pressure-support breath is larger than the $V_{T}$ of the first volume-control breath. This problem may be corrected by decreasing the peak flow in the volume-control breaths. This problem may also occur in a pressure-support breath if the rise time is set too high. An alarm signaling an elevated airway pressure may sound as well. The patient may not want such rapid flow and it may result in active exhalation or coughing.

\section{Cycling Asynchrony}

Cycling refers to termination of ventilator-assisted inspiration. A patient's inspiratory effort may still be present at the time of termination of assisted inspiration. This termination of assisted breathing despite the patient's continued effort is referred to as premature cycling (the reference point is the patient and not the ventilator). The opposite problem may also occur, and is termed delayed cycling: an assisted breath and resultant inspiratory flow may continue into a patient's expiratory effort.

In a spontaneously breathing patient, delayed cycling is marked by lack of patient effort prior to the end of ventilatorassisted inspiration or by the patient actively exhaling during ventilator-assisted inspiration. ${ }^{23}$ Active exhalation can be observed by examination and palpation of the patient's abdomen. Delayed cycling may result in insufficient expiratory time and/or large $\mathrm{V}_{\mathrm{T}}$, with resultant air-trapping, and subsequent ineffective triggering. The pressure-time waveform displays a tenting at the end of ventilator-assisted inspiration (Fig. 7). In pressure-targeted breaths, flow may level off, and this can be detected by examination of the flow-time waveform (Fig. 8). If a patient is actively exhaling, the flow-time waveform may display negative flow. If the ventilatorassisted inspiration is sufficiently out of proportion to patient inspiration, the patient may attempt to take a second breath during ventilator-assisted inspiration. This asynchrony is managed by decreasing inspiratory time and/or $\mathrm{V}_{\mathrm{T}}{ }^{24}$ For patients who have variable inspiratory times, pressure support may be a good option. This is because a pressure-support breath terminates when the flow reaches a certain percentage of peak flow.

Premature cycling is present when a patient is continuing to inhale after ventilator-assisted inspiration has terminated.

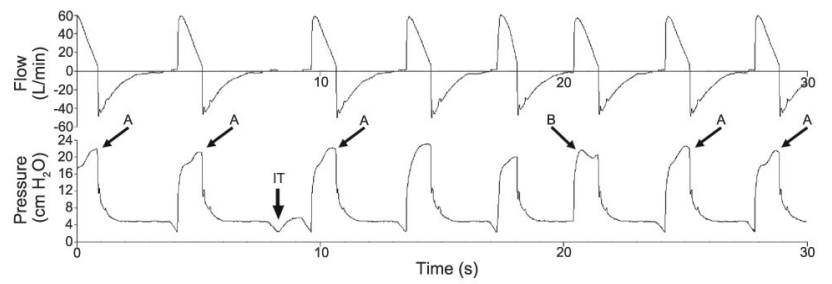

Fig. 7. The peak flows $(60 \mathrm{~L} / \mathrm{min})$ and flow patterns are the same for all the breaths. The tidal volume (not shown, but set to $600 \mathrm{~mL}$ ) is identical for all breaths. This patient is being ventilated with volume-control continuous mandatory ventilation. The peak pressures differ. Breaths denoted by the arrows A show peaking at the end of ventilator-assisted inspiration, suggesting that the patient is no longer inspiring. Note that the breath marked by arrow $B$ is not patient-initiated and that the peak pressure occurs at the beginning of the breath; the pressure generated by patient inspiratory muscle activity is not "pulling down" the pressure curve, and the ventilator is forcing gas in faster than the patient is "pulling it in," resulting in a peak of airway pressure early in the inspiratory cycle. There is also one ineffective effort (arrow IT). The PEEP is $5 \mathrm{~cm} \mathrm{H}_{2} \mathrm{O}$. The patient is ventilated with volume-control continuous mandatory ventilation, with a volume of $600 \mathrm{~mL}$, a peak flow of $60 \mathrm{~L} / \mathrm{min}$, and a decelerating pattern.

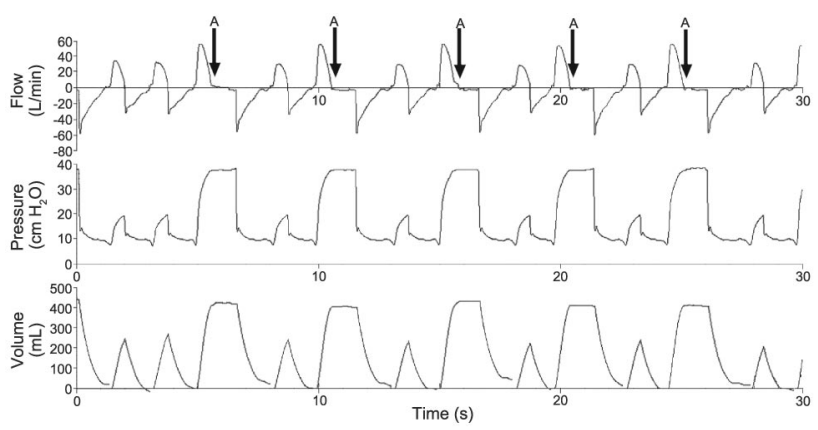

Fig. 8. The patient is being ventilated with 2 types of breaths. In the breaths with the higher peak pressure, higher peak flow, and larger tidal volume, the flow returns to zero half way into mechanical inspiration. The patient has stopped inspiration but the ventilator is continuing to deliver pressure. Pressure equilibration has been reached (arrows A) and no further flow is delivered. This patient is being ventilated with synchronized intermittent mandatory ventilation and pressure support. The PEEP is $10 \mathrm{~cm} \mathrm{H}_{2} \mathrm{O}$. The mandatory breaths (arrows A) are pressure-control breaths $\left(28 \mathrm{~cm} \mathrm{H}_{2} \mathrm{O}\right.$ delivered for $1.70 \mathrm{~s}$ ), resulting in a peak pressure of $38 \mathrm{~cm} \mathrm{H}_{2} \mathrm{O}$. The pressure-support breaths $\left(10 \mathrm{~cm} \mathrm{H}_{2} \mathrm{O}\right)$ have a cycling sensitivity that can be computed graphically: the flow ceases when it is a quarter of the peak flow, indicating that the cycling sensitivity is $25 \%$. The PEEP is $10 \mathrm{~cm} \mathrm{H}_{2} \mathrm{O}$. The patient is ventilated with synchronized intermittent mandatory ventilation and pressure support.

The patient is not exhaling when ventilator-assisted inspiration has ceased. The pressure-time waveform may manifest a decrease in airway pressure, and the flow-time waveform may manifest a simultaneous relative increase in air flow immediately after termination of ventilator-assisted inspiration; finally, the volume-time waveform reveals that gas is 


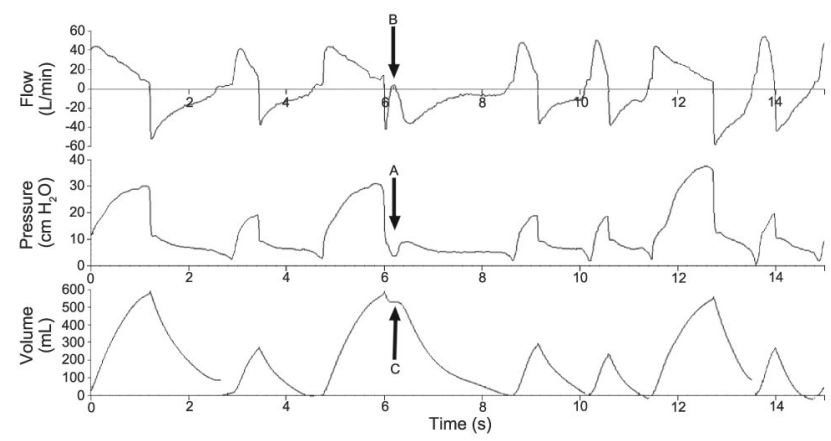

Fig. 9. The third breath has a negative deflection (ie, below PEEP) at the end of the mechanical breath (arrow A) associated with a flow increase (arrow B), indicating that the patient has not ceased to inhale when ventilator-assisted inspiration has ceased. Note the plateau in the volume-time waveform (arrow C) demonstrating that the patient is keeping the volume in their thorax and is not exhaling. This patient is being ventilated with 2 types of breaths. The mandatory synchronized intermittent mandatory ventilation breaths are volume-controlled $(600 \mathrm{~mL})$ and flow-targeted $(50 \mathrm{~L} / \mathrm{min})$. The non-mandatory breaths are pressure-support breaths $\left(15 \mathrm{~cm} \mathrm{H}_{2} \mathrm{O}\right)$ with cycling sensitivity of $25 \%$ (graphically the flow ceases at a quarter of peak flow). The PEEP is $5 \mathrm{~cm} \mathrm{H}_{2} \mathrm{O}$, giving a peak pressure of $20 \mathrm{~cm} \mathrm{H}_{2} \mathrm{O}$ on the pressure-support breaths.

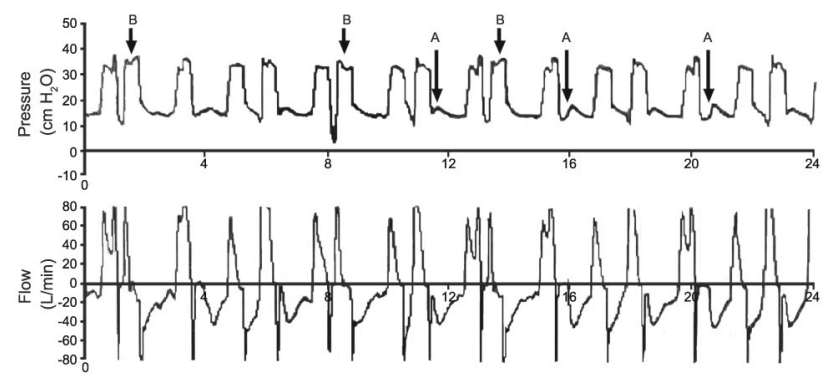

Fig. 10. This figure displays pressure versus time in the top graph and flow versus time in the bottom graph. The patient is experiencing premature cycling (arrows A) and double-triggering (arrows $\mathrm{B}$ ). Some prematurely cycled breaths are more subtle and not marked with arrows (immediately after mechanical breaths 3 , $5,9,12,14,15$, and 17).

not being released from the thorax (Fig. 9). Although this asynchrony meets the definition of ineffective triggering (decrease in airway pressure associated with a flow increase), the timing of the event (immediately after termination of ventilator-assisted inspiration) indicates that the patient is still generating relatively negative intrathoracic pressures with persistent respiratory effort immediately after cessation of assisted inspiration. If patient effort reaches the trigger threshold, the patient will re-trigger the ventilator and another ventilatorassisted breath will be delivered, resulting in double-triggering. The waveform will have the appearance of "bigeminy" or "trigger, trigger, pause" (Fig. 10). For example, if a patient is being ventilated with volume-control ventilation with a $\mathrm{V}_{\mathrm{T}}$ of $450 \mathrm{~mL}$, then the inhaled volume during patient inspiration is $900 \mathrm{~mL}$, not $450 \mathrm{~mL}$. The exhaled $\mathrm{V}_{\mathrm{T}}$ displayed on the initial breath will be $0 \mathrm{~mL}$ and on the second breath will be $900 \mathrm{~mL}$.

Double-triggering is of particular importance in patients with acute respiratory distress syndrome or acute lung injury, because of the desire to avoid large $\mathrm{V}_{\mathrm{T}}$, and has been shown to be more common in volume-control continuous mandatory ventilation. ${ }^{13,25}$ In such a situation the clinician may believe the patient is receiving $6 \mathrm{~mL} / \mathrm{kg}$ ideal body weight, but in actuality the patient may be receiving $12 \mathrm{~mL} / \mathrm{kg}$ ideal body weight. It is only by diligent inspection of exhaled $V_{T}$ and waveforms that the problem is detected. Typically these patients have a high respiratory drive and high minute ventilation. One solution to the problem is to increase the ventilator rate, thereby increasing the mandatory minute ventilation and decreasing air hunger.

\section{Summary}

In conclusion, the patient-ventilator interaction should not impose excessive WOB on the patient. The aim of patient-ventilator synchrony is to achieve synchrony between patient and ventilator during all phases of respiration, including breath initiation, delivery, termination, and exhalation. Ineffective triggering is the most common asynchrony in patients undergoing invasive mechanical ventilation. Flow asynchrony and cycling asynchrony can also be detected by simultaneous examination of patient breathing (ie, facial expressions, mouth breathing, accessory muscle use, and active exhalation with contraction of abdominal muscles) and waveforms displayed on the ventilator.

\section{ACKNOWLEDGMENTS}

Thanks to Thomas C Iden MD for his helpful suggestions and editorial assistance.

\section{REFERENCES}

1. MacIntyre NR, Branson RD. Mechanical ventilation, 2nd edition. St Louis: Saunders Elsevier; 2009.

2. Hess D, Kacmarek RM. Essentials of mechanical ventilation. New York: McGraw-Hill (Health Professions Division); 1996.

3. Brochard L, Slutsky AS. Mechanical ventilation. Update in intensive care medicine series. Berlin Heidelberg: Springer-Verlag; 2005.

4. Tobin MJ. Principles and practice of mechanical ventilation, 2nd edition. New York: McGraw-Hill; 2006.

5. Chao DC, Scheinhorn DJ, Stearn-Hassenpflug M. Patient-ventilator trigger asynchrony in prolonged mechanical ventilation. Chest 1997;112(6): 1592-1599.

6. Giannouli E, Webster K, Roberts D, Younes M. Response of ventilatordependent patients to different levels of pressure support and proportional assist. Am J Respir Crit Care Med 1999;159(6):1716-1725.

7. Parthasarathy S, Jubran A, Tobin MJ. Assessment of neural inspiratory time in ventilator-supported patients. Am J Respir Crit Care Med 2000; 162(2 Pt 1):546-552.

8. Ranieri VM, Grasso S, Mascia L, Martino S, Fiore T, Brienza A, Giuliani R. Effects of proportional assist ventilation on inspiratory muscle 
effort in patients with chronic obstructive pulmonary disease and acute respiratory failure. Anesthesiology 1997;86(1):79-91.

9. Spahija J, de Marchie M, Albert M, Bellemare P, Delisle S, Beck J, Sinderby C. Patient-ventilator interaction during pressure support ventilation and neurally adjusted ventilatory assist. Crit Care Med 2010; 38(2):518-526.

10. Imanaka H, Nishimura M, Takeuchi M, Kimball WR, Yahagi N, Kumon K. Autotriggering caused by cardiogenic oscillation during flowtriggered mechanical ventilation. Crit Care Med 2000;28(2):402-407.

11. Chatburn RL, Primiano FP Jr. A new system for understanding modes of mechanical ventilation. Respir Care 2001;46(6):604-621.

12. Esteban A, Anzueto A, Frutos F, Alia I, Brochard L, Stewart TE, et al; Mechanical Ventilation International Study Group. Characteristics and outcomes in adult patients receiving mechanical ventilation: a 28-day international study. JAMA 2002;287(3):345-355.

13. Thille AW, Rodriguez P, Cabello B, Lellouche F, Brochard L. Patientventilator asynchrony during assisted mechanical ventilation. Intensive Care Med 2006;32(10):1515-1522.

14. de Wit M, Pedram S, Best AM, Epstein SK. Observational study of patient-ventilator asynchrony and relationship to sedation level. J Crit Care 2009;24(1):74-80.

15. Vignaux L, Vargas F, Roeseler J, Tassaux D, Thille AW, Kossowsky MP, et al. Patient-ventilator asynchrony during non-invasive ventilation for acute respiratory failure: a multicenter study. Intensive Care Med 2009;35(5):840-846.

16. Thille AW, Cabello B, Galia F, Lyazidi A, Brochard L. Reduction of patient-ventilator asynchrony by reducing tidal volume during pressuresupport ventilation. Intensive Care Med 2008;34(8):1477-1486.

17. Leung P, Jubran A, Tobin MJ. Comparison of assisted ventilator modes on triggering, patient effort, and dyspnea. Am J Respir Crit Care Med 1997;155(6):1940-1948.
18. Appendini L, Purro A, Patessio A, Zanaboni S, Carone M, Spada E, et al. Partitioning of inspiratory muscle workload and pressure assistance in ventilator-dependent COPD patients. Am J Respir Crit Care Med 1996;154(5):1301-1309.

19. Giuliani R, Mascia L, Recchia F, Caracciolo A, Fiore T, Ranieri VM. Patient-ventilator interaction during synchronized intermittent mandatory ventilation: effects of flow triggering Am J Respir Crit Care Med 1995;151(1):1-9.

20. Ranieri VM, Mascia L, Petruzzelli V, Bruno F, Brienza A, Giuliani R. Inspiratory effort and measurement of dynamic intrinsic PEEP in COPD patients: effects of ventilator triggering systems. Intensive Care Med 1995;21(11):896-903.

21. Marini JJ, Smith TC, Lamb VJ. External work output and force generation during synchronized intermittent mechanical ventilation: effect of machine assistance on breathing effort Am Rev Respir Dis 1988;138(5): 1169-1179.

22. MacIntyre NR, McConnell R, Cheng KC, Sane A. Patient-ventilator flow dyssynchrony: flow-limited versus pressure-limited breaths. Crit Care Med 1997;25(10):1671-1677.

23. Jubran A, Van de Graaff WB, Tobin MJ. Variability of patient-ventilator interaction with pressure support ventilation in patients with chronic obstructive pulmonary disease. Am J Respir Crit Care Med 1995;152(1): 129-136.

24. Tokioka H, Tanaka T, Ishizu T, Fukushima T, Iwaki T, Nakamura Y, Kosogabe Y. The effect of breath termination criterion on breathing patterns and the work of breathing during pressure support ventilation. Anesth Analg 2001;92(1):161-165.

25. Pohlman MC, McCallister KE, Schweickert WD, Pohlman AS, Nigos CP, Krishnan JA, et al. Excessive tidal volume from breath stacking during lung-protective ventilation for acute lung injury. Crit Care Med 2008;36(11):3019-3023.

\section{Discussion}

Hess: A form of asynchrony that I refer to sometimes, that I don't think has come up yet in the discussions here, is "mode asynchrony," which is asynchrony as a result of the mode that we set on the ventilator. I think some of your illustrations are good examples of that with SIMV [synchronized intermittent mandatory ventilation]. Asynchrony can occur because, in SIMV, the ventilator delivers volume-targeted mandatory breaths and pressure-targeted spontaneous breaths.

Branson: It's actually funny to have you here to do this, because we were in this same room [at a previous Journal Conference] with Scott [Epstein] and Dr [Curtis] Sessler and I asked, "Do you guys use SIMV?" and they said, "No, all those patients are on assist-control."
Epstein: We don't use SIMV in my unit.

de Wit: You know, it's an institutional culture, or tradition. People are hesitant to use assist-control. SIMV and pressure support are the 2 most commonly used modes.

Branson: I have no problem with anybody using IMV [intermittent mandatory ventilation] in the right patient. In a patient who comes from the trauma bay after a massive fluid resuscitation and blood products, and he's breathing 26 times a minute and he really just needs $15 \mathrm{~cm} \mathrm{H}_{2} \mathrm{O}$ of PEEP, I have no problem with him being on IMV at a rate of 4 breaths a minute, which is what IMV was designed to do. When John Downs hears people talk about having someone on an IMV rate of 26 he goes insane; he's trying to figure out why anyone would ever do that.

de Wit: That's right. I think the closest we get to assist-control is IMV at 26 breaths a minute.

Branson: On the other hand, I think IMV plus pressure support is often a bad mode for patients with COPD, as Dean suggests, because of the ineffective triggers and the difference between pressure and volume. You use the $\mathrm{Pu}-$ ritan Bennett 840 ventilator. What's your flow waveform during volume control?

de Wit: It's a descending ramp.

Branson: OK. When I first started looking at it and saw those decelerating waveforms, I was afraid it was Volume Control Plus or one of the adaptive modes. 
de Wit: I did have one Volume Control Plus waveform in there.

Branson: What we see all the time is patients in IMV who have just come out of the operating room and are on a rate of 12 breaths a minute, and they're not really breathing, and they start to wake up and the therapists insist on pressure support because you can't breathe with an endotracheal tube without it. Although I continue to remind them that in my first 15 years working there we didn't even own a ventilator that had pressure support, and we actually did send patients home. Then they will decrease the rate, and as the patient becomes more tachypneic, they increase the pressure support. And I'll show up at the bedside and the pressure of the mandatory breath is half the pressure of the pressure-support breath.

Hess: You're producing mode asynchrony!

de Wit: That brings up an important issue, which is the institutional culture of mechanical ventilation and how to standardize it across institutions. I think we all have an institutional culture of mechanical ventilation at our hospitals, and I don't know how to address that problem.

Branson: I agree. Last week there were a couple of times we had patients on IMV of 18 breaths a min, and neither had taken a breath in 2 days! Many people don't want to use assist-control because it's supposedly bad for the respiratory muscles, but they'll set IMV at such a high rate. Neil [MacIntyre] and I have this discussion all the time. I think you could travel the country and give a lecture every day in every city in America about mechanical ventilation, and by the time you were done you'd have to start all over again.

On another topic, is premature valve opening the same as saying the inspiratory time is too short?

de Wit: Yes.
Branson: So it's not that there's something really premature happening, that wasn't expected: it's just that the inspiratory time is too short.

de Wit: Well, it's premature for the patient.

Branson: Right. This younger group of kids are much better visually than they are with numbers. With the emergency-department and anesthesia and surgery fellows I often ask, what's normal compliance, and how do you calculate it? Last time I did that, Bill [Hurford] was there. What's the normal FRC [functional residual capacity]? Anybody? And Bill knows exactly what the lung volumes are. Do they even teach that in medical school anymore? These kids are much better at visually seeing a problem than figuring it out based on the normal value and how to calculate it.

de Wit: The biggest problem now, with so many patients on contact precautions, is getting the whole ICU [intensive care unit] team to enter the patient's room and look at the ventilator graphics. However, by the end of the first week, most house staff can recognize pressure support versus SIMV plus pressure support versus the rare assistcontrol, and they'll recognize ineffective triggers. They are allowed to use the color codes on the Puritan Bennett 840 . I am always impressed by how rapidly they learn to interpret waveforms.

Gentile: Rich, why would you put that patient on a rate of 4 breaths a minute if they're already breathing at 26 breaths a minute? Why not just find something that makes them happy and gives them a reasonable tidal volume? If they're at 26 , why not just let them breathe at 26 ?

Branson: If my patient's problem is hypoxemia-in the old days we'd support that patient with just CPAP and just let them breathe spontaneously, and if you give them a couple breaths per minute, you still end up giving them a slightly larger tidal volume than they'd breathe on their own, and they're very comfortable. Don't do this to a COPD or asthma patient, but if you treat trauma patients on a regular basis, most of the time, unless they have chest-wall deformity, intra-abdominal hypertension, or a head injury, they'll breathe perfectly fine on their own spontaneously, even with no pressure support, and be comfortable and do fine.

Kacmarek: I don't see the rationale for doing that. I think sometimes you program the residents and everybody else to accept that a ventilation mode is useful and appropriate, but then it gets extended to patients in whom it won't work. If you're going to provide ventilatory support, I agree, why not provide it every breath at an appropriate level that assists the patient and unloads their effort instead of forcing the patient to do work periodically and then periodically give them a huge breath. It just makes no sense to me to provide assisted ventilation and then not assist every breath the patient takes.

Parthasarathy: Imsand et $\mathrm{al}^{1}$ had a paper in Anesthesiology, in which they measured the diaphragmatic EMG [electromyogram] signal during IMV, and there was no difference in patient work of breathing between the mandatory and spontaneous breaths. That was, in my opinion, a very eye-opening study.

1. Imsand C, Feihl F, Perret C, Fitting JW. Regulation of inspiratory neuromuscular output during synchronized intermittent mechanical ventilation. Anesthesiology 1994;80(1):1322 .

Epstein: At a low SIMV rate.

Kacmarek: But it was much higher than 4 breaths a minute. You couldn't tell the difference between unassisted and assisted breaths. When you looked at esophageal pressure change and at EMG activity in that paper you could not differentiate the 2 breath types un- 
less you could see the airway pressure curve. The efforts were equivalent.

Parthasarathy: And the second part of it is from the weaning literature. I think that between the 3 large $\mathrm{RCTs}^{1-3}$ there may be still controversy as to what is the method to wean patients (ie, pressure-support weaning or spontaneous breathing trials) and which one is actually capable of taking the patient off faster. But there is no doubt that SIMV is the slowest of the lot. My concern is that, by continuing to use a certain ventilation mode that we know is defective, or in that it is defective only at a lower set respiratory rate, is going to pose difficulties. Moreover, when you switch from a mode used for resting the patient to a mode used for weaning, that poses additional difficulties. You encounter a situation when you say to yourself, "Today we're going to go from the support mode to the weaning mode." That is not really a smooth transition in a given patient, and my concern is that providers become comfortable with one mode that is not really effective for the entire duration of that patient's mechanical ventilation. I think we should shy away from modes that work only in one situation or scenario, and embrace modes that work in all stages of ventilation.

The patient demographics in a trauma ICU are quite different than those in a medical ICU. You could argue that the patients got off the ventilator despite your doing that to them.

1. Esteban A, Frutos F, Tobin MJ, Alía I, Solsona JF, Valverdú I, et al. A comparison of four methods of weaning patients from mechanical ventilation. Spanish Lung Failure Collaborative Group. N Engl J Med 1995; 332(6):345-350.

2. Esteban A, Alía I, Gordo F, Fernández R, Solsona JF, Vallverdú I, et al. Extubation outcome after spontaneous breathing trials with $\mathrm{T}$-tube or pressure support ventilation. The Spanish Lung Failure Collaborative Group. Am J Respir Crit Care Med. 1997; 156(2 Pt 1):459-465.

3. Esteban A, Alía I, Tobin MJ, Gil A, Gordo F, Vallverdú I, Blanch L, Bonet A, Vázquez A, de Pablo R, Torres A, de La Cal MA, Macías S. Effect of spontaneous breathing trial dura- tion on outcome of attempts to discontinue mechanical ventilation. Spanish Lung Failure Collaborative Group. Am J Respir Crit Care Med 1999 Feb;159(2):512-518.

Branson: So the first question is, why would we ventilate somebody who doesn't need to be ventilated, if the patient really just needs PEEP, CPAP, however you want to look at it, and you added a large volume - that's the old days. I think you have to look at IMV now in the context of a low tidal volume, as opposed to large tidal volume of, say, $15 \mathrm{~mL} / \mathrm{kg}$. And I agree that we should never just say that one mode is good and apply it to everybody, but it doesn't matter which mode you pickwhether it's IMV, APRV [airway pressure release ventilation], assist-control, or PAV [proportional assist ventilation]: if you apply them all in the right patients half the time and then just say, "If it's good for half of them, it must be good for all of them"...

Epstein: I want to emphasize that in the weaning literature it was SIMV alone, and here we're talking about SIMV plus pressure support, which is a completely different beast that probably isn't algorithmically different from assist-control, at least if you look at workof-breathing studies, some of which were done by the Tobin group. So we have to make that distinction, I don't think anybody would be advocating for SIMV alone. I want to make sure that the readers understand that we're not talking about that.

Branson: I've been telling people for 20 years that medical ICU patients are different than surgical ICU patients. Twenty years ago we were doing mask CPAP of $10 \mathrm{~cm} \mathrm{H}_{2} \mathrm{O}$ and people said you can't do that: it's dangerous; they'll aspirate; they won't tolerate the mask; it just delays intubation. Today we all strap the mask on the face and put the pressure at $25 \mathrm{~cm} \mathrm{H}_{2} \mathrm{O}$ with a PEEP of $8 \mathrm{~cm} \mathrm{H}_{2} \mathrm{O}$, and we think we're geniuses! Time always brings these things out. I think that IMV is terribly inappropriate in a patient who has ventilatory muscle failure. In a patient with hypoxemia who, until they got shot, stabbed, or hit by a car had perfectly normal respiratory mechanics, if you provide the right amount of PEEP you'll find that they breathe reasonably well on any kind of spontaneous mode. We see it in the literature: APRV, IMV, PAV, or pressure support.

Hurford: I can't understand why you want to support different breaths differently. I don't see the advantage of more asynchrony in breathing, when the topic of the conference is that we can't get people to breathe correctly. How does adding additional types and forms of breathing and additional different inspiratory times help? It's not surprising that patients are asynchronous: they're confused! They don't know what breath to take next. What's the rationale for that with the microprocessor ventilators we have now?

Kacmarek: There's no rationale.

Hurford: Rich, why IMV?

Branson: Again, I don't know if I'm advocating for IMV. I always tell people in the ICU, if you decided today that we had to put everybody on assist-control, I could live with that. If you decided today that we had to put everybody on SIMV, I could live with that. The important thing is setting the ventilator appropriately for the patient. I see these studies time after time in which COPD patients in a medical ICU are put on SIMV, and it's confusing to me. But I've seen lots of patients with hypoxemic respiratory failure- not ventilatory failure-do perfectly fine breathing spontaneously with a couple of interspersed breaths.

Hurford: That's because they don't need a ventilator.

Branson: I agree. So that's the whole concept, right? Does the patient with hypoxemic respiratory failure need a ventilator or do they just need CPAP? 
MacIntyre: I don't like IMV, for the reasons that several of you have said. I think that the patient's respiratory controller does get confused, and I think the reason the inspiratory efforts are the same is because the patient doesn't know what's going to come next and can't adapt to it. I think that leads to asynchrony, and during my presentation I'm going to show some data that support that concept.

With that in mind, let me comment on the mode that I like to use. I admit that I have a medical ICU, so it may not apply to surgeons, but I like the pressure assist-control mode. It gives you a couple of interesting features. For example, it gives you a backup rate if you want it, and if you don't want it, you can turn the rate down to one or less on most ventilators, in which case it becomes very much like pressure support, because it's patient-triggered and pressure-targeted. The only difference is that it is time-cycled. Your controlled breaths and your machine breaths actually look very similar to the patient. And depending on what level of pressure you apply, you can titrate, if you will, the amount of work a patient's going to do during the assisted breaths. It takes away the need for IMV because you have the backup rate and it provides the advantage of pressure-targeting for most of the patient's breaths. I'm just throwing that out as a mode that I know a lot of people don't use, but perhaps it is underused.

Gentile: Rich, I'm sorry: I didn't mean to jump on you and then have everybody else jump on you. Talking about institutional culture, in 1989 I presented grand rounds on a patient who was had failed to wean in a thoracic unit and was transferred to our medical unit. We had her on IMV and pressure support, and I was harshly criticized for this very reason: why is the patient on 2 breath types? And slowly but surely I saw all the other staff leave the room, run up to the ICUs, and change all the ventilators to eliminate the set rates. And that was the last time we put anybody on a mixed mode. So that's our institutional culture: if there's a reliable trigger, we don't set a rate. Right or wrong, that's the way it works. This makes it easy for training, and some literature indicates that healthy volunteers say that this mode feels more comfortable than that mode, when you go back and forth.

Parthasarathy: I think Bill [Hurford] asked the rationale for giving 2 different kinds of breaths. Correct me if I'm wrong, but pressure support came about because in the old Servo 900 ventilator you couldn't actually give a higher flow rate if you had them on assist-control, so you had to change it to target the minute volume. In that quirky little ventilator, the flow rate was a function of where you set the minute ventilation and machine set rate. That's how this ventilation mode came into being! It was essentially a quirk of a machine that made us want to give two different breaths, and it stuck with us even though the new ventilators had new physiologically based modes, designed by physiologists. I think we should be using those physiologically based modes and do away with those older ways of ventilating.

Kallet: I think that's part of the story. Another part of it had to do with trigger work, and we started adding low-level pressure support, then people started turning up the pressure support so the pressure-support tidal volumes and pressures were higher than IMV: it became crazy. Part of this is just culture. We used to have tails: we got rid of the tails, but we still use IMV.

I came from an IMV school in upstate New York, and they were total fanatics about IMV in the late 1970s. The problem was that with really bad trauma patients with severe ARDS [acute respiratory distress syndrome] we would paralyze them and set an IMV rate of 18 breaths a minute, and the IMV flow would wash through the circuit. But if you talk to anyone, "No, you have to have spontaneous breathing," but when patients are that sick, we do CMV [con- tinuous mandatory ventilation] until we can stabilize the patient. Probably everyone does that, but when we talk ideologically about what we'd prefer, then we start talking past one another.

Branson: I'm just trying to respond to the fact that everyone seems to think that IMV is bad, and I never go to the medical ICU unless they ask me. It appears to me that IMV is definitely bad for their patients, but I think other patients do perfectly well on IMV: those who have hypoxemic respiratory failure. I agree.

Where Bill [Hurford] and I work it's not unusual to find patients so sedated they couldn't possibly breathe and they're still on IMV. I recoil at the fact that it can have a negative connotation in the wrong patient, but I don't think it's always negative every time people use it. In some of the earlier IMV weaning trials they only dropped the rate 2 breaths a minute every day, so IMV took longer than everything else. The people who designed IMV, their concept was that weaning initiated the second you put them on the ventilator. Bob Kacmarek knows this: he used to work with Shapiro and all those guys.

Kacmarek: John Downs was there at Northwestern the first time I set up IMV. But remember, at the time we introduced IMV, the Emerson was a very commonly used ventilator. We had ventilators that could only provide control mode ventilation. Few ventilators allowed assisted ventilation at that time, and IMV allowed patients to breathe spontaneously, thus improving synchrony. Part of the reason it became part of the culture was because it really served a genuine need at the time because of the ventilators that were available.

Pierson: One thing apparent to me from this discussion is that there's a lack of equipoise about this issue, even among the experts in this room. If you expand that not only to around the United States but also throughout the world-as has been shown in several studies ${ }^{1-4}$ 
it's clear that an objective view of modes of mechanical ventilation is not presently at hand. Clinicians generally have their minds made up about which modes are preferable, although there is no consensus, and the opinions tend to be held pretty strongly. I know of a major United States university medical center, an ARDS Network participant, at which, because of overflow problems, a medical ICU patient landed in the surgical ICU and was put on volume assist-control, whereupon the respiratory therapists (who were unit-based) were so scandalized that they actually put in for an ethics consult-because of the use of a mode that was at variance with their culture. We're dealing with the facts here at the conference, and trying to sort out what's going on with patient-ventilator interaction and see if we can make sense of it or say anything for sure. We're going to have to deal with this incredible lack of equipoise about the issue on the part of the highly divergent cultures even within institutions.

1. Esteban A, Anzueto A, Alía I, Gordo F, Apezteguía C, Pálizas F, et al. How is mechanical ventilation employed in the intensive care unit? An international utilization review. Am J Respir Crit Care Med 2000; 161(5):1450-1458.

2. Rose L, Presneill JJ, Johnston L, Nelson S, Cade JF. Ventilation and weaning practices in Australia and New Zealand. Anaesth Intensive Care 2009;37(1):99-107.
3. Koh Y, Lim CM, Koh SO, Ahn JJ, Kim YS, Jung BH, et al. A national survey on the practice and outcomes of mechanical ventilation in Korean intensive care units. Anaesth Intensive Care 2009;37(2):272-280.

4. Metnitz PG, Metnitz B, Moreno RP, Bauer P, Del Sorbo L, Hoermann C, et al; SAPS 3 Investigators. Epidemiology of mechanical ventilation: analysis of the SAPS 3 database. Intensive Care Med 2009;35(5):816-825.

Younes:* Going back to graphics, I want to ask a provocative question. How confident are you by looking at the ventilator waveforms that your interpretation is correct?

de Wit: You can't be $100 \%$ sure.

Younes: There are so many things that can look like ineffective efforts that are not ineffective efforts. Your comment that only $1 \%$ of breaths have a prolonged inspiratory phase is at total variance with my own observations of the diaphragm, in which most breaths had a marked difference between the end of inspiratory effort and the end of the ventilator cycle. So I would suggest caution about relying completely on the waveforms.

de Wit: I agree. However, having esophageal balloons in all patients is not

\footnotetext{
* Magdy Younes MD FRCP(C) PhD, Department of Medicine, University of Manitoba, Winnipeg, Manitoba, Canada.
}

feasible. There needs to be a balance between invasive monitoring and waveform interpretation.

Epstein: In my talk I mentioned that this is the tip of the iceberg, and we really don't know. Marjolein, you said that ineffective efforts were the most common type of asynchrony, but I think that depends on the patient population. I think double-triggering is more common in acute lung injury, and cycling problems is the big one in noninvasive ventilation. It really depends on the patient population.

Younes: But also in pressure-support ventilation in the ICU. I've done lots of measurements of this, and if you compare the end of diaphragmatic pressure to the end of the ventilator breath, this is a very common problem.

Epstein: Right, but it's difficult to detect that noninvasively. You're detecting it with an esophageal balloon.

Younes: I'm just saying that you have to be careful about concluding that there's no expiratory asynchrony just from the waveforms, without some additional means.

This article is approved for Continuing Respiratory Care Education credit. For information and to obtain your CRCE

(free to AARC members) visit

RCJournal.com 